\title{
Fàbregas et al Rehabilitation method affects behavior, welfare, and adaptation potential for subsequent release of orphaned white rhinoceros
}

\author{
Peter K. McGregor ${ }^{1}$ • Sophie L. Mowles ${ }^{2}$ \\ Published online: 14 October 2020 \\ (C) ISPA, CRL 2020
}

The reason for this editorial comment is that the ms raised a number of issues during the reviewing process that could apply to rehabilitation studies of captive animals of conservation concern.

The purpose of this editorial comment is two-fold.

The first is to reiterate the indicative nature of the results. Two features of the study would ordinarily mean it would not be published in the journal. However, lack of replication (there was one hands-on and one hands-off facility) and non-independence of subjects because of group housing, both arise as a consequence of the practicalities of reacting to a conservation crisis rather than poor experimental design. It seems highly unlikely that either feature could be changed in the future because the demands of practical conservation solutions must be of more importance than experimental design.

The second purpose is to explain why, these issues notwithstanding, we considered publication could nonetheless contribute to developing best practice in applied behavior related to conservation. We decided to accept the ms in part because the authors address each issue as far as is possible in the ms, but mainly because publication is a way to make widely available information that could potentially change rehabilitation approaches in many species of conservation concern. A subsidiary purpose is to seek the views of others on the general question of publishing indicative results from studies with an applied aim, where circumstances mean that there will always be limited scope for experimental design (particularly replication and subject independence). We are particularly interested in how institutions / facilities dealing with circumstances similar to those of rhino orphans seek to disseminate developing best practice. To this end, we invite comments via the journal's Facebook page.

Publisher's note Springer Nature remains neutral with regard to jurisdictional claims in published maps and institutional affiliations.

See related article, https://doi.org/10.1007/s10211-020-00343-w

Peter K. McGregor

pkmcgregor@ispa.pt

Sophie L. Mowles

sophie.mowles@anglia.ac.uk

1 ISPA - Instituto Universitário, Lisbon, Portugal

2 Anglia Ruskin University, Cambridge, UK 